\title{
肌酸激酶变性过程中圆二色光谱变化 及旒基暴露的速度
}

姚启智 梁树坚 田敏 邹承鲁*

(中国科学院生物物理研究所, 北京)

摘要

本文以 $\mathrm{CD}$ 光谱及疏基测定两种方法监测了肌酸激酶的变性过程. 快速可反应 疏基的测定表明，颈基暴露速度与从前报道过的荧光光谱及紫外差光谱的变化速度 大体相当。但是,在低浓度胍溶液中,隐蔽的芳香氨基酸尚未暴露时, 酶分子的梢圆 度值已有了明显可察的变化. 变性速度的比较表明, $220 \mathrm{~nm}$ 平均残基陌圆度值的变 化速度明显地慢于芳香氨基酸、巯基的暴露速度. 这可能意味着,肌酸激酶分子内某 些肽段具有松散的二级结构, 又靠近分子表面,易受到低浓度胍的变性作用。而整个 酶分子的二级有序结构的破坏, 还是慢于卷曲肽链的伸展速度, 也即三级结构的破坏 速度。

酶的变性与失活的研究是探讨酶的三维结构与催化功能之间关系的重要手段, 这方面早 已引起相当的注意. 但是将变性动力学与失活动力学作详细地比较研究者不多. 关于肌酸激 酶的情况, 也大体如此. Dawson ${ }^{[1]}$, Price ${ }^{[2]}$ 以及 Grossman ${ }^{[3]}$ 等人曾先后报道了肌酸激酶的变 性是可逆的、充分复原的酶与天然酶的性质十分相近、变性酶的复活情况在相当范围内与酶的 浓度无关等等. 我们于前文 ${ }^{[4-5]}$ 中详细地报道了肌酸激酶在不同浓度胍中的变性速度与失活 速度的比较,其结果是变性速度总是明显地慢于失活速度.特别是在低胍浓度下更是如此. 考 虑到酽的三维结构是异常复杂的,变性时的构象变化更是景象万千,任何一种监测手段所获结 果都有其片面性. 我们在前面工作中所采用的紫外差光谱及苂光光谱两种方法, 监测的结果 仅仅是反映了一些与色氨酸、酪氨酸侧链基团相关的构象变化. 为了更全面地了解变性过程 中酶分子的构象变化情况，显然应该从多种角度进行监测. 本文即以圆二色光谱（以下简称 $\mathrm{CD}$ 谱)变化及琉基的暴露速度两种方法进一步探测肌酸激酶经胍、朓变性时的变性速度.

显然, 酶分子变性时, 肽链主链的构象变化也是很重要的方面. CD 谱是监测蛋白质分子 二级结构变化的有力手段. 有关蛋白质及多肽经胍、腿变性后的 $\mathrm{CD}$ 谱的研究, 报道甚多 ${ }^{[6,7]}$. 1981 年, Price ${ }^{[2]}$ 等在作变性酶的复性复活时, 将充分复活的肌酸激酶的 $\mathrm{CD}$ 谱与天然酶的 $\mathrm{CD}$ 谱做过比较. 但是, 以 $\mathrm{CD}$ 谱追踪肌酸激酶的变性情况, 监测其变性速度的报道, 尚未见到. 我们在本工作中,除测定了不同变性剂浓度下变性酶的 $\mathrm{CD}$ 谱外, 还在固定波长下, 监测了酶

本文 1983 年 8 月 31 日收到, 1984 年 4 月 11 日收到修改稿.

* 本文部分工作是由邹承鲁在美国哈佛医学院生物化学生物物理医学中心(波荻顿)任访问教授时所做的工作. 
变性动力学过程.

变性过程中，侧链基团的暴露速度同样能从另一个侧面反映出构象变化速度. 肌酸激酶 分子内有 8 个琉基,链间没有二硫键. Kuby ${ }^{[8]}$ 等人曾以 Ellman 的 DTNB 法测得天然肌酸激 酶分子内有两个可反应琉基,经 $8 \mathrm{M}$ 㾇素变性后,每一酶分子可测得 6 个可反应放基, 因此他 们曾推测尚有一对放基可能处于具有明显负电荷的微环境中，DTNB 分子不易接近. 可以预 期,随着肽链的不断伸展,隐蔽的琉基会不断地暴露. 在本工作中，我们连续监测了酶在变性 过程中，可反应䘪基数目的增加速度. 并将此放基暴露速度与其它方法监测到的变性速度加 以比较.

\section{一、材 料 与 方 法}

肌酸激酶按 Kuby B 法自兔骨骼肌内提取 ${ }^{[9]}$, 再以 DEAE-纤维素进一步纯化, 经凝胶电 泳鉴定为一条区带。制备方法及比活力测定等均按前文 ${ }^{[4]}$ 中所述方法进行.

胍基盐酸盐按 Nozaki ${ }^{[10]}$ 法重结晶 2 次,腺素经 $95 \%$ 、无水乙醇重结晶 2 次. DTNB 试剂 [5.5' 二硫代-双 (2-硝基苯甲酸)]系 BDH 产品. 其它试剂均为北京化工厂产品,分析纯.

圆二色光谱的测定应用 Cary 61 型圆二色光谱仪,于 $25^{\circ} \mathrm{C}$ 下, 在 $200-260 \mathrm{~nm}$ 远紫外区 测定. 溶液体系为 Tris-缓冲体系, $\mathrm{pH} 8.3$; 酶浓度为 3.4-136 $\mu M$. 对于不同酶浓度样品 采用不同光径的比色池，以保证椭圆度读数值在同样范围内. 在测定不同胍浓度下变性酶的 $\mathrm{CD}$ 谱时,一般采用 $0.1 \mathrm{~mm}$ 光径的比色池, 以使在短波范围内胍的吸收减到最小限度. 胍溶 液中的 $\mathrm{CD}$ 谱均经同样浓度胍的校正. 变性动力学数据, 是在 $220 \mathrm{~nm}$ 固定波长下, 扫描记录其 $\theta$ 值随时间的变化. 所得之数据按一级反应动力学作图法, 以 $\ln \frac{\theta_{t}-\theta_{\infty}}{\theta_{0}-\theta_{\infty}}$ 对时间 $t$ 作图, 从 得到的直线关系中求得变性速度常数.

可反应琉基数目的测定是用 Ellman 的 $\mathrm{DTNB}^{[11]}$ 法, 具体做法是在每 $1 \mathrm{ml}$ 待测酶溶液 中 (5.6 $\mu M$, 甘氨酸缓冲体系, $\mathrm{pH} 9.0)$, 加人 $10 \mu \mathrm{l} 0.1 \mathrm{M}$ DTNB 溶液, 于 $412 \mathrm{~nm}$ 波长下, 读 取吸收值, 采用消光系数 $13600 \mathrm{M}^{-1} \mathrm{~cm}^{-1}$, 计算出相应的琉基的克分子浓度, 对应于酶浓度, 即可求出每一酶分子具有的可反应放基数目.

当测定天然酶与充分变性酶的琉基数目时发现, 加人 DTNB 试剂 $20 \mathrm{~s}$ 后, $412 \mathrm{~nm}$ 处光吸 收值已经不再随时间改变. 故放基暴露速度测定是在酶变性后, 间隔时间取样, 加人 DTNB 后, 用 Cary 219 双光束分光光度计的恒温自动连续采样装置, 将反应液葲人流通池内 (thermostatable routine sampler); 第一点数据是酶加变性剂加 DTNB 后 20 s 时读数, 其余各点也是 于不同的变性时间间隔取样后, 加入 DTNB，20 s 时于 $412 \mathrm{~nm}$ 波长下读取光吸收值. 对照光 路上, 放置同样浓度的 DTNB 溶液, 用以校正 DTNB 的本底吸收和因自行水解造成的吸收.

\section{二、结 果}

\section{1. 不同浓度胍溶液中变性酶的 CD 谱}

充分变性的肌酸激酶的远紫外 CD 谱已经为 Price 等人 ${ }^{[2]}$ 报道过. 本工作详细测定了自 $0.3 \mathrm{M}$ 至 $3.0 \mathrm{M}$ 胍溶液中变性酶的 $\mathrm{CD}$ 谱. 结果表明, 即使在 $0.3 \mathrm{M}$ 胍溶液中, 肌酸激酶的 远紫外 $\mathrm{CD}$ 谱已经有了明显可察的变化, 且随着胍浓度的升高, $220 \mathrm{~nm}, 208 \mathrm{~nm}$ 两处负峰值 
明显降低. 胍浓度高于 $1 M$ 时,酶的二级结构受到了很大的破坏,在 $3 M$ 时. 由于胍在远紫外 区域的强吸收的干扰，即使用 $0.1 \mathrm{~mm}$ 的比色池，也无法测定高浓度胍溶液中酶的远紫外 CD 谱. 所获测定结果见图 1 .

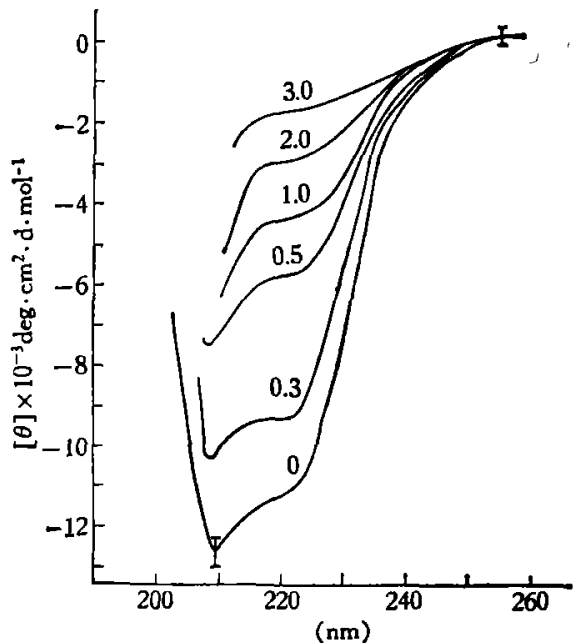

图 1 肌酸激酶在不同浓度胍溶液中变性 $\mathrm{CD}$ 谱 [酶浓度: $0.034 \mathrm{mM}$, tris-缓冲体系, $\mathrm{pH} 8.3,25^{\circ} \mathrm{C}$ 变性 $18 \mathrm{hr}$, 图中数字表示胍浓度 $(M)]$

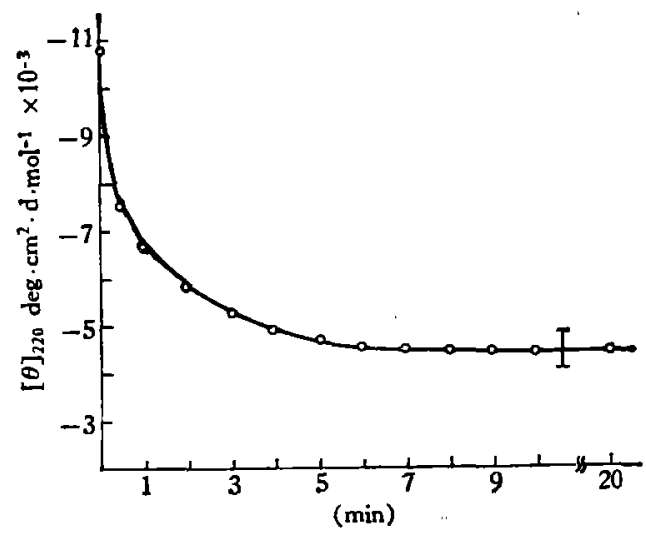

图 2 肌酸激酸在 $1 M$ 胍中变性时椭圆度变化速度

（酶浓度: $0.034 \mathrm{mM}$, 肌: $1 M$, tris-缓冲体系 $\mathrm{pH}$ $8.3,25^{\circ} \mathrm{C}$ 下,自变性开始连续监测 $220 \mathrm{~nm} \theta_{\mathrm{obs}}$ 值的变化）

表 1 肌酸激酶在胍溶液中变性速度与失活速度的比较

\begin{tabular}{c|c|c|c|c|c|c}
\hline 变 性 剂 & \multicolumn{5}{|c|}{ 变速度常数 $\mathrm{s}^{-1} \times 10^{3}$} & \multicolumn{2}{|c}{ 失 活 } \\
\cline { 2 - 7 } 胍 $(M)$ & 紫外差光谱 & 苂光光谱 & $\mathrm{CD}$ 光谱 & $-\mathrm{SH}$ 暴露速底 & $\begin{array}{c}\text { 速度常数* } \\
\mathrm{s}^{-1} \times 10^{3}\end{array}$ & 剩余活力\% \\
\hline 0.3 & $-\mathrm{a}$ & $-\mathrm{a}$ & $-\mathrm{c}$ & $-\mathrm{c}$ & $-\mathrm{c}$ & 40 \\
& $-\mathrm{a}$ & $-\mathrm{a}$ & 0.39 & 1.2 & 1.5 & 30 \\
0.5 & $-\mathrm{a}$ & $-\mathrm{a}$ & $-\mathrm{c}$ & $-\mathrm{c}$ & 3600 & 15 \\
& 4.3 & 3.6 & 0.77 & 1.4 & 3.1 & 3.4 \\
1.0 & 53 & 40 & 17 & $-\mathrm{b}$ & 4300 & 0 \\
3.0 & 1900 & $-\mathrm{c}$ & $-\mathrm{b}$ & $-\mathrm{b}$ & 5900 & 0 \\
\hline
\end{tabular}

* 一级反应速度常数: 一 $\mathrm{a}$ 一未见明显可察变化，一b一反应太快未能测定，一c一未未测。

\section{2. 由 CD 谱的变化测定酶的变性速度}

在不同浓度胍溶液中,肌酸激酶的变性速度测定，是在 $220 \mathrm{~nm}$ 固定波长下监测变性酶的 椭圆度值随时间的变化. $1 \mathrm{M}$ 胍溶液中酶的 $\theta_{220}$ 值随时间的变化速度如图 2 所示. 在更高浓 度胍溶液中,由于其变化速度过快, 无法以 $\mathrm{CD}$ 谱直接追踪. 所得之各动力学曲线, 按一级动 力学作图法求得其速度常数. 详细结果列于表 1 .

值得注意的是, 尽管在 $0.3 \mathrm{M}$ 胍变性时, $\mathrm{CD}$ 谱已有了明显变化, 但是它的变化速度却比 $1 M$ 胍变性时的速度慢了两个数量级之多。

\section{3. 蛋白浓度对变性酶 CD 谱的效应}

在以前的工作中 ${ }^{[4-5]}$, 我们观察到肌酸激酶在胍溶液中变性时, 失活速度远快于变性速度 


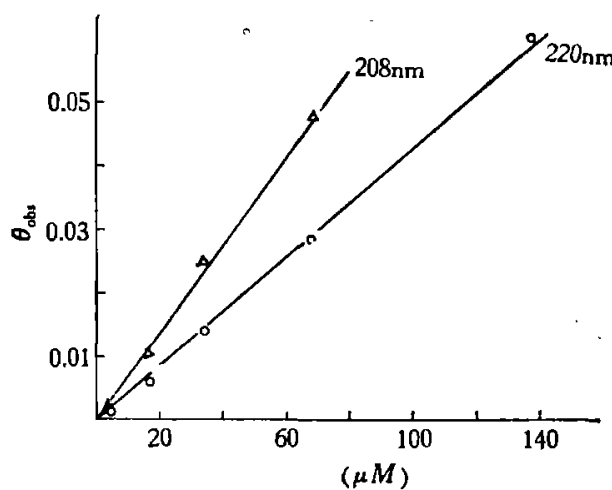

图 3 酶浓度对肌酸激酶变性时椭圆度值 $\left(\theta_{\text {obs }}\right)$ 的影响

[酶浓度: $(0.034-0.136 \mathrm{mM})$, tris-缓冲体系 $\mathrm{pH}$ $8.3,25^{\circ} \mathrm{C}$, 曲线 1 和 2 分别代表不同浓度酶变性后 的 $220 \mathrm{~nm}$ 和 $208 \mathrm{~nm}$ 的 $\theta_{\mathrm{obs}}$ 值]
表 2 肌酸激酶在胍溶液中变性时疏基的暴露，

\begin{tabular}{|c|c|c|}
\hline 胍浓度 $(M)$ & $\begin{array}{c}\text { 可反应 SH } \\
\text { 数目/酶分子 }\end{array}$ & $\begin{array}{l}\text { 暴露速度 } \\
\mathrm{s}^{-} \times 10^{3}\end{array}$ \\
\hline 0 & 1.70 & -- \\
\hline 0.3 & 3.19 & 1.23 \\
\hline 0.5 & 6.74 & 1.39 \\
\hline 1.0 & 6.89 & nd* \\
\hline 3.0 & 6.78 & $\mathrm{nd}^{*}$ \\
\hline
\end{tabular}

*反应过快,未能测定.

为了探讨快速失活是否与酶分子的双体解聚 有关, 我们测定了自 3.4-136 $\mu M$ 范围内不 同浓度酶在 $1 M$ 胍溶液中 CD 谱（不同浓度 酶测定时，采用了不同光径的比色池）。将 $208 \mathrm{~nm}, 220 \mathrm{~nm}$ 两个波长下测得椭圆度值分别对应于酶浓度作图,得到了很好的直线关系. 其 情形如图 3 所示. 说明在此酶浓度范围内，变性酶的平均残基椭圆度值与酶浓度无关.

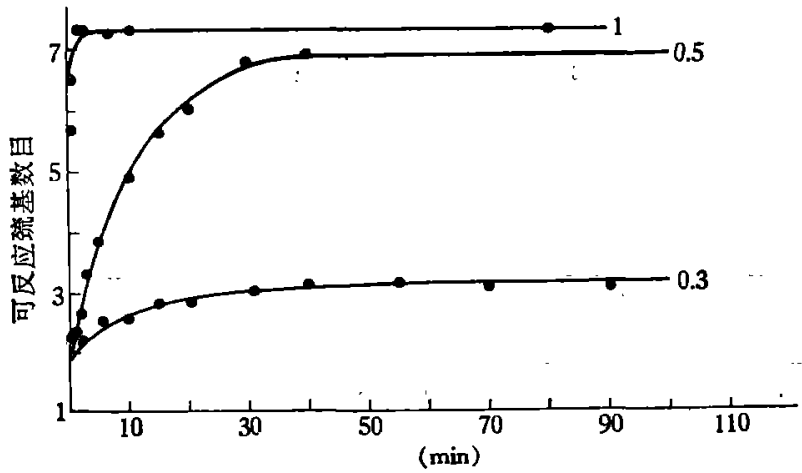

图 4 肌酸激酶在不同浓度胍溶液中变 性时巯基暴露速度

[酶浓度: $0.0056 \mathrm{mM}$, 甘氨酸缓冲体系 $\mathrm{pH} 9.0,25^{\circ} \mathrm{C}$, DTNB 法测定琉基 (洋见材料与方法部份) 图中数字代表

表 3 肌酸激酶在腿溶液中变性时 颈基的暴管

\begin{tabular}{c|c|c}
\hline 胍浓度 $(M)$ & $\begin{array}{c}\text { 可反应琉基 } \\
\text { 数目/酶分子 }\end{array}$ & $\begin{array}{c}\text { 暴䈃速度* } \\
\mathrm{s}^{-1} \times 10^{3}\end{array}$ \\
\hline 1.70 & - \\
\hline 1.0 & 1.77 & - \\
\hline 2.0 & 1.96 & - \\
\hline 3.0 & 5.52 & 0.41 \\
\hline 4.0 & 6.59 & 3.83 \\
\hline 5.0 & 6.78 & 5.75 \\
\hline
\end{tabular}

* 一级反应速度常数. 胍浓度 $(M)]$

在同样的酶浓度范围内 $(3.4-136 \mu M)$, 测定了不同浓度酶在 $1 M$ 胍溶液中的变性速度， 结果表明,在此范围内, 变性速度同样与酶浓度无关.

此外,我们还测定了不同 $\mathrm{pH}$ 条件下的 $\mathrm{CD}$ 谱, 结果表明, 在 $\mathrm{pH} 7.11-8.58$ 范围内酶的 $\mathrm{CD}$ 谱不受 $\mathrm{pH}$ 值的影响.

\section{4. 变性酶放基暴露数目的测定}

肌酸激酶于不同浓度胍、脲溶液中充分变性 $(24-48 \mathrm{~h})$ 后, 可反应琉基数目的测定结果列 于表 2,3 中.

我们的测定是在 $\mathrm{pH} 9.0$ 甘氨酸缓冲体系中进行, 对比实验表明, 所获结果与 $\mathrm{pH} 8.0$ Tris 缓冲体系所获结果是一致的. 为排除 $\mathrm{pH} 9.0$ 下 DTNB 可能自行水解造成的干扰, 每次测定 
均在对照光路上同时放置相同体系相同浓度的 DTNB 溶液作为对照.

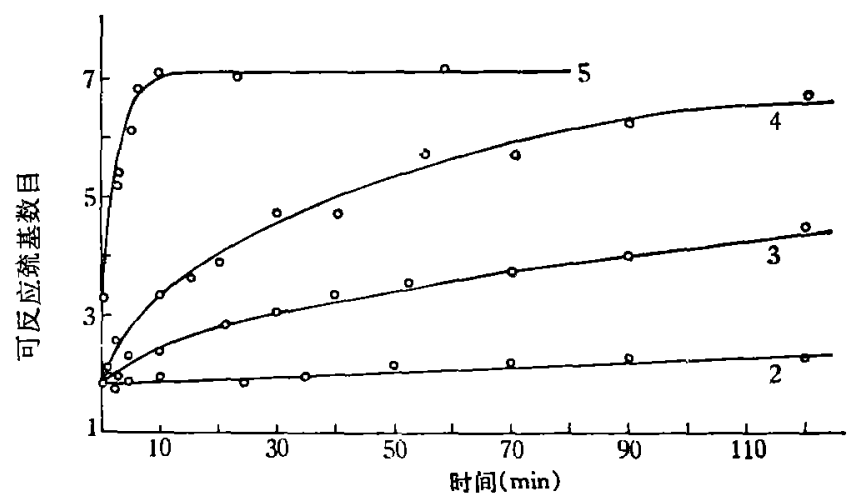

图 5 肌酸激酸在不同浓度豚溶液中变性时琉基暴籍速度 [除变性.剂为䐂外,其它条件同于图 4. 图中数字代表豚浓度 $(M)]$

当测定天然酶与充分变性酶的 疏基数目时, $20 \mathrm{~s}$ 后, $412 \mathrm{~nm}$ 光吸收 值即不再随时间改变. 但对 $0.3 \mathrm{M}$ 胍和 $3 M$ 嫝中变性 $24 \mathrm{~h}$ 的酶来说， 加人 DTNB 后, $412 \mathrm{~nm}$ 光吸收值 仍然出现随时间而缓慢变化, 经数 小时后，最终达到充分变性酶的数 值 (6个琉基)为止. 对于这一过程, 可能有两种解释. 其一是 $0.3 \mathrm{M}$ 胍 和 $3 M$ 腿变性时得到的是一种部份 变性态. Tiffany 等人 ${ }^{[6]}$ 称此为局部 松散的螺旋结构 (Local extended helix structure). 像 DTNB 这种小 分子除立即修饰其充分暴露的表面琉基外, 还可能逐步向松散了的分子内部扩散, 逐渐接近 那些内部的巯基,其接近程度, 由分子的松散程度所决定。第 二种解释则是部份变性态是由 完全变性的分子和完全未变性 的分子形成某种平衡态，当 DTNB 分子修饰了一个疏基 后,便会影响了上述平衡,很可 能导致变性酶分子数目的进一 步增加, 可反应巯基数目也随 之增加. 直至所有酶分子全变 性, 所有可测得的统基全暴露 为止. 但是, 无论是上述两

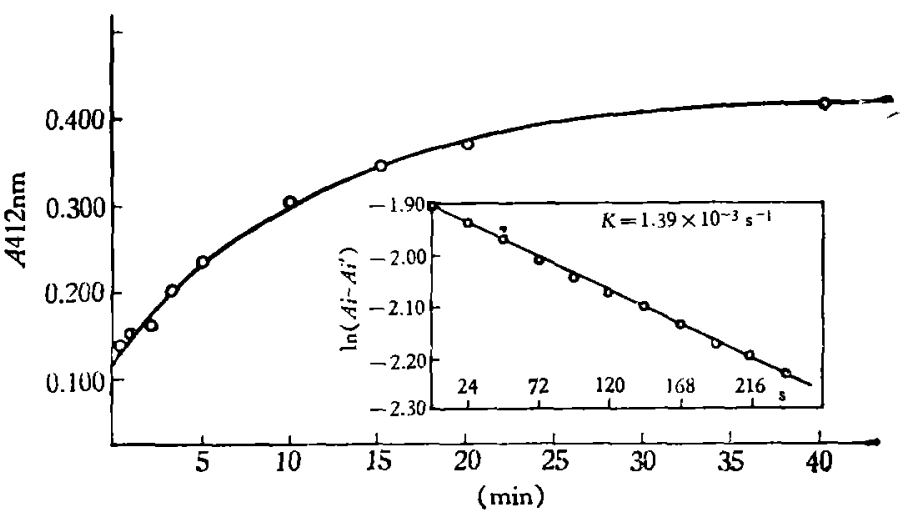

图 6 巯基暴露速度的一级动力学常数作图 (条件同于图 4, 变性剂为 $0.5 \mathrm{M}$ 胍, 图中内插图为 Guggenhim 作图求其速度常数)

种可能的那一种情形, 加人 DTNB 试剂 $20 \mathrm{~s}$ 后, $412 \mathrm{~nm}$ 光吸收值的进一步增加, 显然不是 DTNB 分子与琉基的反应时间所致.

\section{5. 放基墨露速度的测定}

测定 0.3-3.0 M 胍溶液及 $1-8 \mathrm{M}$ 腿溶液中酰变性过程中巯基暴露速度的结果分别如图 4 及图 5 所示. 由图中可见,胍的变性作用远强于腿的作用. $1 M$ 胍溶液中的放基暴露速度已 经明显地快于 $5 \mathrm{M}$ 腿溶液变性时的情形。根据巯基暴露的动力学曲线, 再按一级反应动力学 Guggenhim 作图法求其速度常数. 图 6 给出了酶于 $0.5 \mathrm{M}$ 胍溶液中变性时放基暴露速度的 Guggenhim 作图. 所获之速度常数也列于表 2,3 中.

\section{三、讨 论}

蛋白质变性包括着寡聚体的解聚,肽链的伸展, 螺旋、折叠等有序二级结构的松散等复杂 
过程. 不同的监测方法只能反映上述构象变化的某一侧面. 荻光光谱、紫外差光谱所反映的 是与酪氨酸、色氨酸残基相关的构象变化, 可反应笁基数目的增加则是某一侧链基团的暴露情 况的标志, 而 $\mathrm{CD}$ 谱的变化反映着肽链主链的伸展状况. 我们的实验结果表明, 当采用这些不 同的方法监测酶的变性过程时, 所反映出的变性程度与变性速度,均有所不同. 在 $0.3 \mathrm{M}$ 胍溶 液中,部份变性酶的 CD 谱有了明显的变化, 可反应琉基数目也由 2 个增加至 4 个, 但是此 时, 苂光光谱及紫外差光谱均未见明显变化. 当胍的浓度增加至 $0.5 \mathrm{M}$, 腿的浓度至 $4 M$ 时, 可反应琉基数自己达最大值, 但紫外差光谱及苂光光谱的变化尚未达到最大值. 这些现象可 能意味着酶分子内某些肽段的二级结构可能裸露于分子表面, 低浓度胍的作用即可使其松散. 同时, 还有两个疏基是处于靠近分子表面及结构不太紧密的肽段上,因而低浓度胍即可与之相 互作用. Terrossian ${ }^{[3] 3}$ Keto $^{[14]}$ 等先后报道他们以含永的巯基修饰剂修饰肌酸激酶的两个疏基 后,还可以继续以磑代乙酰胺等试剂再修饰两个琉基,由此他们认为,酶分子尚有两个“隐蔽較 基”十分靠近天然酶的活性部位放基, 在适宜的条件下, 含永修饰剂可于巯基间发生转移. 这 一推测,与我们观察到的实验现象也颇为一致. 但是遗憾的是肌酸激酶的氨基酸序列至今仍 属未知, 否则我们就可在低浓度胍溶液中, 将变性酶标记上 DTNB 分子, 再根据其氨基酸序列 特征，从而区分开那一段肽段对低浓度胍作用是敏感的.

表 4 肌酸激酶在腿溶液中变性速度常数与失活速度常数的比较

\begin{tabular}{|c|c|c|c|c|c|}
\hline 变性剂 & \multicolumn{3}{|c|}{ 变性速度常数* $\mathrm{s}^{-1} \times 10^{3}$} & \multicolumn{2}{|c|}{ 失 } \\
\hline 腿 (M) & 紫外差光谱 · & 苂光光谱 & 矮基暴露 & $\begin{array}{c}\text { 速度常数* } \\
\mathrm{s}^{-1} \times 10^{3}\end{array}$ & 剩余活力\% \\
\hline 1.0 & $-a$ & -2 & $-a$ & 4000 & 59 \\
\hline 2.0 & $-a$ & $-\mathbf{a}$ & $-\mathbf{a}$ & 7000 & 32 \\
\hline 3.0 & 0.24 & 0.24 & 0.41 & 10000 & 14 \\
\hline 4.0 & 0.32 & 0.60 & 3.83 & 13000 & 5 \\
\hline 5.0 & 2.86 & 1.02 & 5.75 & $-b$ & 0 \\
\hline
\end{tabular}

* 一级反应速度常数, 一 $-\mathrm{a}$ 一未见明显可察变化, 一b一未测.

表 1, 4 中列出了各种不同方法所测得的变性速度常数. 比较这些速度常数可知, 不同方 法所得之速度常数有着明显可见的差异. 正如所预期的那样, 紫外差光谱及苂光 光谱所测 得的速度常数是相同的. 琉基暴露速度略慢于这两者的速度，而 CD 谱的变化速度则更慢. Tiffiny 等人认为蛋白质经胍、胒变性后, 其二级结构并不一定呈完全无规卷曲状态, 很可能是 一种局部松散了的蠌旋状态. 本实验室尚未发表的工作也表明肌酸激酶经低浓度十二烷基硫 酸锂变性后, 活性已丧失, 紫外及茨光光谱均有了明显变化后, 但其 CD 谱未见明显变化.

表中列出的各种方法测得的变性速度均明显地慢于相同条件下酶的失活速度. 在低浓度 胍 $(0.3-0.5 \mathrm{M})$ 溶液中, 失活呈两相, 快相失活大约占总活力的 $80 \%$, 其失活速度是相应的 变性速度的 1000 倍左右. 慢相失活的速度与变性速度大体相同．在高浓度胍 $(1.0-3.0 \mathrm{M})$ 和腿溶液中, 失活速度仍然明显快于变性速度. 对于这些现象的可能有三种解释. 其一是由 于变性剂胍与肌酸的结构有相似之处, 胍有可能作为一个可逆竞争性抑制剂与酶相作用, 而 
引起快速失活, 但是, 当以豚作变性剂时, 同样出现快速失活, 这就基本上排除了胍的可逆抑制 作用. 其二是低浓度胍、腿变性时,很可能会引起肌酸激酶二聚体的解聚. 曾有人报道过酶在 低浓度胍作用下发生解聚 ${ }^{[1] 7}$, 但是肌酸激酶变性时的解聚情况还不清楚. 该酶单体是否有活 性的问题,目前也未有定论. Price ${ }^{[18]}$ Grossman ${ }^{[3]}$ Nevinsky ${ }^{[19]}$ 等先后报道, 认为其亚基可能有 活性. Degani ${ }^{[20,21]}$ 等根据肌酸激酶分子内两个亚基上的疏基被修饰时, 表现出非均一性, 从 而推测两个亚基的结构与功能也会不均一. 我们的实验结果表明, 在 40 倍浓度范围内, 失活 与变性的速度与酶浓度无关, 同时, 也在数十倍范围内, 复性复活的速度也与酶浓度无关 ${ }^{[22]}$, 这 些都表明,快速失活不大可能是由于解聚引起的. 最后, 快速失活的最可能的原因便是酶的活 性部位具有着非常严格的空间结构, 胍与腿这些变性剂分子很容易与酶的活性部位相作用, 在 整个酶分子发生明显的构象变化之前, 活性部位已经首先遭受破坏, 从而表现为快速的失活过 程. 许多晶体衍射的结果也表明,活性部位仅仅是酶分子内很有限的一个区域 ${ }^{[23-25]}$,酶的快速 失活也可能是由于酶的活性部位的构象完整性是依赖于整个酶分子构象的完整性, 甚至发生 于距离活性部位较远的区域的构象变化,也会闶即传递至活性部位,使得酶快速失活.

\section{参考文献}

[ 1 ] Dawson, D. M. et al., J. Biol. Chem., 242(1967), 210.

[ 2 ] Bickerstaff, G. F. et al., Biochim. Biophys. Acta., 621(1980), 305.

[ 3 ] Grossman, S. H. et al., Biochemistry, 20(1981), 6122.

[4] 姚启智等,中国科学 B 辑, 1982,10: 892 .

[5] 姚启智等,中国科学 B 輯, 1982,12: 1084 .

[ 6 ] Chen, Y. H. et al., Biochemistry, 11(1972), 4120.

[ 7 ] Balasubrannian, D., Biopolymers 13(1974), 407.

[ 8 ] Yue, R. H. et al., Biochemistry, 6(1967), 3204.

[ 9 ] Noda, L. et al., Methods in Enzymeol. (Ed. Colowick, S. P. and Kaplan, N. O.), Acad. Press, New Youk, Vol 2, 1954, 605 .

[10] Nozaki, Y., ibid., 26(1972), 43.

[11] Ellman, C. L., Arch. Biochem. Biophys., 82(1959), 70.

[12] Goggenheim, E. A., Phil. Mag., 1926, 2: 538.

[13] Der Terrossian, E. and Kassab, R., Eur. J. Biochem., 70(1976), 623.

[14] Fawcett, A. H. et al., Biochem. Biophys. Res. Commun. 107 (1982), 302

[15] Tiffany, M. L. and Krimm, S., Biopolymers, 12(1973), 575.

[16] Zou, C. L. et al., (Unpublish).

[17] Edelstein, C. and Scanu, A. M., J. Biol. Chem., 255(1980), 5747.

[18] Bickerstaff, G. F. and Price, N. C., FEBS lett., 64(1976), 319.

[19] Nevinsky, G. A., et al., FEBS LETT., 149(1982), 36.

[20] Degani, C. and Degani, Y., J. Biol. Chem., 255(1980), 8221.

[21] Degani, C. and Degani, Y., Biochemistry, 18(1979), 5917.

[22] 侯立向等,生物化学与生物物理学报, 15(1983), 393.

[23] Blake, C. C. F. et al., Proc. Roy. Soc., B167(1967), 378.

[24] Moult. J. et al., J. Mol. Biol., 100(1976), 179.

[25] Liljas, A. et al., Nature, New Biol. (London), 235(1972), 131 